\title{
High Grade Myeloid Neoplasm
}

National Cancer Institute

\section{Source}

National Cancer Institute. High Grade Myeloid Neoplasm. NCI Thesaurus. Code C150122.

A finding indicating that a myeloproliferative process has high-grade pathologic and/or clinical features. 